had antimicrobial susceptibility testing (AST) to determine minimum inhibition concentrations (MICs) for Cefixime (CFM), Ceftriaxone (CRO), Azithromycin (AZI), Gentamicin (GEN), and Ciprofloxacin (CIP) using E-test.

Results From November 2015-August 2016, 900 specimens were collected; 713 (79.2\%) specimens were from $\mathrm{BH}$ and 187 (20.8\%) were from SCC. Among the 900 specimens, 479 (53.3\%) had NG growth; 478 (99.8\%) NG isolates had AST performed. Seventeen men had repeat NG infections. Among the 461 men with at least one infection, 291 (63.1\%) had sex with women only, 138 (29.9\%) had antibiotic use in the last 2 weeks, and all received treatment for gonorrhoea. The median age of men with NG infection was 29 years (range 14-76 years). All NG isolates were susceptible by Clinical and Laboratory Standards Institute standards to CFM, CRO, AZI and GEN; 438 of $478(91.6 \%)$ isolates were resistant to CIP. Conclusion We report the first 10 months of data from EGASP Thailand. Most isolates were found to be susceptible to all tested antibiotics except CIP. Surveillance is critical to assess trends and risk factors for NG, and to monitor for emergence of resistance.

\section{P3.66 HOW FAR IS THE FIGHT AGAINST HBV AND HIV AMONG ANTENATAL ATTENDEES IN MAKURDI METROPOLITAN CITY, BENUE STATE, NIGERIA}

${ }^{1}$ Emmanuel Msugh Mbaawuaga, ${ }^{1}$ Atakpa $\mathrm{Pb},{ }^{2}$ rroegbu Cu, ${ }^{3} \mathrm{kke} \mathrm{Ac}$. 1. Department of Biological Sciences, Benue State University Makurdi, Makurdi, Nigeria, Nigeria; ${ }^{2}$ Department of Biological Sciences, Cross River University of Technology Calabar, Calabar, Nigeria; ${ }^{3}$ Department of Microbiology, University of Nigeria Nsukka, Nsukka, Nigeria, Nigeria

\subsection{6/sextrans-2017-053264.301}

Introduction: Benue State in Nigeria is one of the regions in sub-Saharan Africa with rising morbidity and mortality among adults from HIV/AIDS and other sexually transmitted diseases. This retrospective study was conducted to determine the impact of the fight against HBV and HIV among antenatal attendees in Makurdi metropolitan city.

Methods A total of 757 cross sectional blood samples; 250 (2007) and 507 (2012) were collected and screened for both Hepatitis B surface antigen (HBsAg) and HIV antibodies. HBsAg was screened using rapid test kits and ELISA kits while HIV antibodies was screened for using Determine (Japan) and Stat Pak (USA) rapid test kits. Other information generated with the help of questionnaire and data analysis was done using chi square in SPSS version 20 software. P values equal or less than 0.05 was considered significant.

Results Overall, HBV infection rate of $12.4 \%$ (2007) was significantly higher $(\mathrm{p}=0.027)$ than $7.5 \%$ in 2012 but there was no difference in HIV rates between 2007 and 2012 $(p=0.777)$. There was also a significant difference in $\mathrm{HBV}$ rates among attendees with no formal education in 2007 $(26.7 \%)$ and those of $2012(8.3 \%)(p=0.029)$. HIV on the other hand was significantly $(\mathrm{p}=0.025)$ lower among individuals with secondary education in 2008 (1.2\%) compared with those of $2012(8.2 \%)$. Farmers (2008) significantly had higher HBV (35.5\%) than their counterpart in $2012(0.0 \%)$ $(p=0.001)$. Similarly, HBV infection rates recorded in 2007 was significantly higher than those of 2012 among those vaccinated, unvaccinated $(p=0.016)$. as well as those with history of transfusion $(p=0.008)$, Alcoholics $(p=0.043)$ and surgery $(\mathrm{p}=0.043)$.
Conclusion The study observed a significant decrease in HBV infection between 2007 and 2012 attributable to HBV vaccination, safer blood transfusion and surgery. Hence, there is need to sustain the fight against HBV but efforts needs to be intensified towards combating HIV.

\section{P3.67 RECRUITMENT AND RETENTION OF CHLAMYDIA TRACHOMATIS POSITIVE WOMEN IN THE MULTICENTER LONGITUDINAL COHORT STUDY FEMCURE}

1,2 L Eppings, ${ }^{1,2} \mathrm{CJPA}$ Hoebe, ${ }^{3} \mathrm{~T}$ Heijman ${ }^{3} \mathrm{AA}$ of Hogewoning, ${ }^{4,5,6} \mathrm{HM}$ Götz, ${ }^{3,7,8,9} \mathrm{HJ}$ de Vries, ${ }^{2}$ PFG Wolffs, ${ }^{1,2} \mathrm{NHTM}$ Dukers-Muijrers. 'Public Health Service South Limburg, Department of Sexual Health, Infectious Diseases and Environmental Health, Geleen, the Netherlands; ${ }^{2}$ Maastricht University Medical Centre - School of Public Health and Primary Care (CAPHRI), Maastricht, the Netherlands; ${ }^{3}$ STI Outpatient Clinic, Public Health Service of Amsterdam (GGD Amsterdam), Amsterdam, The Netherlands; ${ }^{4}$ Department Infectious Disease Control, Municipal Public Health Service Rotterdam-Rijnmond (GGD Rotterdam), Rotterdam, The Netherlands; ${ }^{5}$ National Institute of Public Health and the Environment (RIVM), Epidemiology and Surveillance Unit, Centre for Infectious Disease Control, Bilthoven, The Netherlands; ${ }^{6}$ Department of Public Health, Erasmus MC - University Medical Centre Rotterdam, Rotterdam, The Netherlands; ${ }^{7}$ Department of Infectious Diseases, Public Health Service of Amsterdam (GGD Amsterdam), Amsterdam, The Netherlands; ${ }^{8}$ Centre for Infection and Immunity Amsterdam (CINIMA), Academic Medical Centre (AMC), Amsterdam, The Netherlands; ${ }^{9}$ Department of Dermatology, Academic Medical Centre, University of Amsterdam, Amsterdam, The Netherlands

\subsection{6/sextrans-2017-053264.302}

Introduction Longitudinal cohort studies provide unique insights but preventing drop-out and missing values is challenging. The aim of this study is to describe how to recruit and maximise retention of 400 Chlamydia trachomatis (CT) positive women in FemCure, an ongoing longitudinal multicenter cohort study aiming to recruit (extra)genital CT positive women.

Methods Recruitment took place at 3 STI-clinics (South Limburg, Amsterdam and Rotterdam). Of the invited women 29\% joined. They collected vaginal, anorectal and (nurse-taken) oral swabs and completed online questionnaires pre-treatment, 1, 2, 4, 6, 8, 10 and 12 weeks post-treatment. To minimise loss to follow up (ltfu) various reminders were sent, small incentives were given and no show at the 2 nd visit were replaced. Logistic regression analyses were used to assess predictors for ltfu (i.e. age, education level, steady partner, ethnicity, previous CT diagnosis, recruitment clinic, and anatomic site of infection). The results include the first 7 months of recruitment.

Results By 11-dec-16 143 participants completed fu. Of them, $81 \%$ had complete data, $17 \%(n=24)$ was ltfu, of which 18 before the 2 nd visit. Nine cases had missing data. Of all questionnaires and swabs $(\mathrm{n}=1144), 12 \%$ was completed after a reminder. Multivariate logistic regression showed that education and age were associated with ltfu. Low education was related to more ltfu than higher education $(\mathrm{OR}=2.8$, 95\% CI 1.1-6.8). Those aged $23=>$ were related to more ltfu than those $<23(\mathrm{OR}=2.5$, 95\% CI 1.0-6.4). No other variables were statistically significantly associated, this may change with ongoing recruitment. At the last visit $86 \%$ reported to participate again in a similar study (reasons given include helping others/friendliness of the staff) while the others mention time constraints as a barrier.

Conclusion These preliminary results show a retention rate of $81 \%$ without missing data. Approaches described, especially combined with committed healthcare providers can be used to motivate participants to complete $\mathrm{fu}$ in future longitudinal multicenter studies. 